\title{
LOWER BOUNDS ON COLORING NUMBERS FROM HARDNESS HYPOTHESES IN PCF THEORY
}

\author{
SAHARON SHELAH
}

\begin{abstract}
We prove that the statement "for every infinite cardinal $\kappa$, every graph with list-chromatic number $\kappa$ has coloring number at most $\beth_{\omega}(\kappa)$ " proved by Kojman [7] using the RGCH theorem [13] implies the WRGCH theorem, which is a weaker relative of the $\mathrm{RGCH}$, via a short forcing argument.

Similarly, a better upper bound than $\beth_{\omega}(\kappa)$ in this statement implies stronger (consistent) forms of the WRGCH theorem, the consistency of whose negations is wide open.

Thus, the optimality of Kojman's upper bound is a purely cardinal arithmetic problem, and, as discussed below, is hard to decide.
\end{abstract}

\section{INTRODUCTION}

Recall that the list-chromatic or choosability number of a graph $G=$ $\langle V, E\rangle$ is $\kappa$ if $\kappa$ is the least cardinal such that for any assignment of lists of colors $L(v)$ to all vertices $v \in V$ such that $|L(v)| \geq \kappa$ there exists a proper vertex coloring $c$ of $G$ with colors from the lists, namely $c(v) \in L(v)$ for all $v \in V$. A graph $G$ has coloring number $\kappa$ if $\kappa$ is the least cardinal such that there exists a well-ordering $\prec$ on $V$ such that a vertex $v \in V$ is joined by edges to only $<\kappa$ vertices $u$ satisfying $u \prec v$.

Alon [1] proved that every finite graph with list-chromatic number $n$ has coloring number at most $(4+o(1))^{n}$ and this bound is tight up to a factor of $2+o(1)$ by [3].

In [7] Kojman used the Revised GCH theorem from cardinal arithmetic [13] to prove in ZFC the upper bound of $\beth_{\omega}(\kappa)$ on the coloring number of any graph with a list chromatic number $\leq \kappa$, where $\beth_{\omega}(\kappa)$ is the cardinal gotten by applying the exponent function to $\kappa$ infinitely many times. ${ }^{1}$

By Erdös and Hajnal [2] from 1966, if the GCH is assumed, $\kappa^{++}=$ $\left(2^{\kappa}\right)^{+}=\left(\beth_{1}(\kappa)\right)^{+}$bounds the coloring number of every graph with listchromatic number $\kappa$ for every infinite $\kappa$. It is now known that much weaker axioms than the GCH - certain weak consequences of the Singular Cardinals Hypothesis - imply the same upper bound (see the second section in

Version 2016-02-18_11. See https://shelah.logic.at/papers/1052/ for possible updates.

The author thanks the Israel Science Foundation for partial support of this research, Grant no. 1053/11. Publication 1052.

${ }^{1}$ Formally, $\beth_{0}(\kappa)=\kappa, \beth_{n+1}(\kappa)=2^{\beth_{n}(\kappa)}$ and $\beth_{\omega}(\kappa)=\lim _{n} \beth_{n}(\kappa)$. 
[7]), so in "many" models of set theory, the upper bound is $\left(2^{\kappa}\right)^{+}$. Komjath [6] recently improved the GCH upper bound to $2^{\kappa}=\kappa^{+}$, constructed models of the $\mathrm{GCH}$ in which $\chi_{\ell}(G)=\operatorname{Col}(G)$ for every graph with infinite $\chi_{\ell}(G)$ and showed that in MA models $2^{\kappa}$ is required.

The sharp contrast between the single exponent in the bound for the finite case, or in the bound for the infinite case in the presence of mild cardinal arithmetic axioms, and the infinite tower of exponents in the ZFC bound, led Kojman to ask whether the upper bound $\beth_{\omega}(\kappa)$ could be lowered in ZFC. He also asked whether the use of the RGCH in proving his $\beth_{\omega}$ bound was necessary.

We prove here that:

(1) Kojman's $\beth_{\omega}$ upper bound implies the so called Weak Revised GCH theorem (WRGCH) in pcf theory.

(2) better upper bounds imply open strengthenings of the WRGCH theorem.

All implications above are via standard forcing arguments.

Thus, improving Kojman's upper bound on the coloring number of a graph in terms of its list-chromatic number will be at least as hard as improving the WRGCH theorem. In particular, a better upper bound cannot be gotten with only graph-theoretic arguments.

Note that consistency results in pcf are hard; only recently Gitik [4] succeed to make a remarkable advance: for a countable set $\mathfrak{a}$ of regular cardinals, $\operatorname{pcf}(\mathfrak{a})$ may be uncountable. Grand as it is, this is a far cry from what is needed to show that the WRGCH cannot be improved. If, however, all relevant strengthenings of the WRGCH are indeed not provable in ZFC, then Kojman's $\beth_{\omega}$ bound is optimal (a more detailed discussion of this is given below).

We thank Menachem Kojman for asking us the questions and for reading two earlier versions of this proof and the present one.

\subsection{Description of the reduction.}

Definition 1.1. The Weak Revised GCH theorem, WRGCH, is the statement that for every strong limit cardinal $\mu>\aleph_{0}$, e.g. $\beth_{\omega}$, and $\lambda>\mu$, for some $\kappa<\mu$ there is no sequence $\bar{\lambda}=\left\langle\bar{\lambda}_{i}: i<\mu\right\rangle$ of finite sequences of regular cardinals in $(\mu, \lambda)$ such that $J_{<\lambda}(\bar{\lambda}) \subseteq[\mu]^{<\kappa}$.

Here, the pcf operator is extended to sets $\mathfrak{a}$ of finite sets (as above, we identify a finite sequence of cardinals with its range) by letting $\operatorname{pcf}(\mathfrak{a})$ be interpreted as $\operatorname{pcf}(\bigcup \mathfrak{a})$ and similarly $J_{\lambda}(\mathfrak{a})$ stands for $\{\mathfrak{b}: \mathfrak{b} \subseteq \mathfrak{a} \& \max \operatorname{pcf}(\mathfrak{b})<$ $\lambda\}$.

The WRGCH is a straightforward consequence of the Revised GCH theorem [13].

Now consider, for a natural number $m \leq 1$, the following two closely related statements (even equivalent, see below) with parameter $m$, which are stronger than the WRGCH: 
$\oplus_{m}^{1}$ there are no cardinal $\kappa$ and set $\mathfrak{a}$ of $\beth_{m}(\kappa)$ many finite sequences of regular cardinals, each larger than $\beth_{m}(\kappa)$, such that $J_{<\sup (\mathfrak{a})}[\mathfrak{a}] \subseteq$ $[\mathfrak{a}]^{<\kappa}$, i.e. $\mathfrak{b} \in[\mathfrak{a}]^{\kappa}$ implies that $\max \operatorname{pcf}(\mathfrak{b}) \geq \sup (\mathfrak{a})$.

$\oplus_{m}^{2}$ there are no cardinals $\kappa$ and $\Upsilon$ satisfying $\partial:=\beth_{m}(\kappa) \leq \Upsilon$ and a family of sets $\mathcal{A} \subseteq[\Upsilon]^{\partial}$ such that $|A \cap B|<\kappa$ for all distinct $A, B \in \mathcal{A}$ and $|\mathcal{A}|>\Upsilon$

The status of the statements above is as follows. If $m<n$ then $\oplus_{m}^{i}$ implies $\oplus_{n}^{i}$ for $i \in\{1,2\}$. All $\oplus_{m}^{i}$ hold in models of the GCH or even of just the strong hypothesis (see [12], §6), so are consistent with the axioms of ZFC.

The question for which $m$ is $\oplus_{m}^{i}$ a theorem of ZFC is wide open, that is, for all $m \geq 1$, neither a ZFC proof nor a consistency of the negation is known at the moment. The WRGCH, however, is a theorem of ZFC, as it follows trivially from the RGCH theorem.

Lowering $\beth_{\omega}(\kappa)$ in Kojman's upper bound to $\beth_{n}(\kappa)$ for some $n<\omega$ is at least as hard as proving the equivalent statements $\oplus_{m}^{1}$ and $\oplus_{m}^{2}$ for $m=2 n+1$. The reason for this is that if the configuration that is forbidden by e.g. $\oplus_{m}^{2}$ does exists in some model $\mathbf{V}$ of ZFC then in some forcing extension of $\mathbf{V}$ there is a graph with list-chromatic number $\theta$ and coloring number $>\beth_{n}(\theta)$, for some $\theta>\kappa$. The relation $m=2 n+1$ can probably be tightened, but we made no effort to do so.

Also, if it is assumed to the contrary that the configuration that is forbidden by the WRGCH does exists in some universe $\mathbf{V}$ of set-theory, then in some forcing extension $\mathbf{V}^{\mathbb{P}}$ of $\mathbf{V}$ there is a graph with list-chromatic number $<\kappa$ and coloring number $>\beth_{\omega}(\kappa)$, contrary to the $\beth_{\omega}$ upper bound. Thus, the $\beth_{\omega}$ graph-theoretic bound implies the WRGCH.

We discuss next the pcf-theoretic statements and explain further their connection to upper bounds on coloring numbers.

Let $\kappa \leq \partial<\Upsilon<\lambda=\operatorname{cf}(\lambda)$ be cardinals. Consider the statement:

$(s t)_{\kappa, \partial, \Upsilon, \lambda}^{1}$ there is a $\mathcal{A} \subseteq[\Upsilon]^{\partial}$ of cardinality $\lambda$ such that if $A_{1} \neq A_{2}$ belong to $\mathcal{A}$ then $\left|A_{1} \cap A_{2}\right|<\kappa$.

We agree that if $\lambda=\Upsilon^{+}$we may omit it and if $\mu=\Upsilon, \lambda=\chi^{+}=\mu^{+}$then we also may omit them, so the typical case $(s t)_{\kappa, \mu}^{1}$ is the existence of a family $\mathcal{A} \subseteq[\mu]^{\mu}$ of cardinality $\mu^{+}$which is a $\kappa$-family, that is, the intersection of any two distinct members of $\mathcal{A}$ has cardinality $<\kappa$.

Why is using $(s t)_{\kappa, \mu}^{1}$ reasonable when $\beth_{m}(\kappa) \leq \mu<\beth_{m+1}(\kappa)$ ? The history of this question is rich. In particular, Baumgartner got by forcing, without using large cardinals, the consistency of $(s t)_{\kappa, \mu}^{1}$ with $\kappa=\kappa^{<\kappa}<\mu<2^{\kappa}$, so here $m=0$.

We are, however, interested in the cases $m \geq 1$, which are closely related to pcf problems.

Consider the pcf statement, 
$(*)_{\kappa, \mu, \chi, \lambda}^{2} \kappa<\mu<\chi<\lambda=\operatorname{cf}(\lambda)$ and there is a sequence $\overline{\mathfrak{a}}=\left\langle\mathfrak{a}_{i}: i<\mu\right\rangle$ of finite sets of regular cardinals with each $\mathfrak{a}_{i} \subseteq(\mu, \chi)$ and such that $\lambda=\max \operatorname{pcf}\left(\bigcup_{i} \mathfrak{a}_{i}\right)$ and $J_{<\lambda}[\overline{\mathfrak{a}}]=\left\{u \subseteq \mu: \operatorname{pcf}\left(\bigcup_{i \in u} \mathfrak{a}_{i}\right) \subseteq \lambda\right\} \quad$ (so really $\chi \gg \mu$. The main case, and the one we shall deal with, for transparency, is $\lambda=\chi^{+}$.)

Why $(s t)_{\kappa, \mu, \chi, \lambda}^{1}$ and $(*)_{\kappa, \mu, \chi, \lambda}^{2}$ are related to each other and to graph colorings?

$(*)_{0}$ if $\mathcal{A} \subseteq[\chi]^{\mu}$ has cardinality $>\chi$, and is a $\kappa$-family, $\kappa \leq \mu \leq \chi$ then the natural bi-partite graph associated to $\mathcal{A}$ and denoted $G_{\mathcal{A}}$, (see definition 2.4 below) has coloring number $\geq \chi^{+}$.

So finding such $\mathcal{A}$ with small list-chromatic number, say $\kappa$, with $\beth_{n}(\kappa) \leq$ $\lambda=\chi^{+}$, will give consistent lower bounds, which is the purpose of this note. The main point here is that the list-chromatic number of such graphs can be lowered by applying the internal forcing axiom from [14] (see also [18]), a natural generalization of MA.

Observe that

$(*)_{1}$ For $\ell=1,2$,

(a) if (st) $)_{\kappa_{1}, \mu_{1}, \chi}^{\ell}$ and $\kappa_{1} \leq \kappa_{2} \leq \mu_{2} \leq \mu_{1}$ then $(*)_{\kappa_{2}, \mu_{2}, \chi}^{\ell}$.

(b) if (st) $)_{\kappa_{1}, \partial_{1}, \Upsilon_{1}, \lambda_{1}}^{\ell}$ and $\kappa_{1} \leq \kappa_{2}<\mu_{2} \leq \mu_{1}$ and $\Upsilon_{1} \leq \Upsilon_{2}<\lambda_{2} \leq \lambda_{1}$ then $(\mathrm{st})_{\kappa_{2}, \partial_{2} \Upsilon_{2}, \lambda_{2}}^{\ell}$.

$(*)_{2}$ For $\ell=1,2$,

(a) (st) $)_{\kappa, \partial, \Upsilon}^{2}$ implies (st) $)_{\kappa, \partial, \Upsilon}$ and (st) $)_{\kappa, \partial, \Upsilon, \lambda}$ implies (st) $)_{\kappa, \partial, \Upsilon, \lambda}$.

(b) If (st) ${ }_{\kappa, \partial, \Upsilon}^{\ell}$ and $\Upsilon=\Upsilon_{1}^{+}, \mu_{1}=\min \left\{\partial, \Upsilon_{1}\right\} \geq \kappa$ (so $\ell=1 \wedge \partial=$ $\Upsilon \Rightarrow \partial_{1}=\partial$ ) then $(\mathrm{st})_{\kappa, \partial_{1}, \Upsilon_{1}}^{\ell}$

(c) If (st) $)_{\mu \kappa, \partial \Upsilon, \lambda}^{\ell}$ and $\partial<\Upsilon$ and $\Upsilon$ is a limit cardinal of cofinality $\neq \operatorname{cf}(\partial)$ and $\neq \operatorname{cf}\left(\lambda\right.$ then for every large enough $\Upsilon_{1}<\Upsilon$ we have $(\mathrm{st})_{\kappa, \partial, \Upsilon_{1}, \lambda}^{\ell}$

Also

$(*)_{3}$ If $\partial^{<\kappa}<\lambda=\operatorname{cf}(\lambda), \kappa=\operatorname{cf}(\kappa)>\aleph_{0}$ and $(\mathrm{st})_{\kappa, \partial, \Upsilon, \lambda}^{1}$ then $(\mathrm{st})_{\kappa, \partial, \Upsilon, \lambda}^{2}$.

Why? By [11], 6.1 with $\lambda, \Upsilon, \partial, \kappa^{*}$ here substituting $\partial^{*}, \partial, \kappa, \sigma$ there. Similarly, by Theorem 6.2 in [11] we have $\oplus_{m}^{1} \Longleftrightarrow \oplus_{m}^{2}$ for all $m \geq 1$.

Let

$(*)_{\kappa, \mu}^{0, n} \mu \in\left(\beth_{n}(\kappa), \beth_{n+1}(\kappa)\right)$.

So the problem with the consistency of $(\mathrm{st})_{\kappa, \mu, \Upsilon}^{1}+(\mathrm{st})_{\kappa, \partial}^{0, n}$ is having $(\mathrm{st})_{\kappa, \partial, \Upsilon}^{2}+$ $(\mathrm{st})_{\kappa, \partial}^{0, n}$.

We may note that clause (b) is justified by the $\mathrm{RGCH}$ and $\lambda=\operatorname{cf}(\lambda)$ is usually natural.

An example, then, of how this note clarifies the question of whether the upper bound of $\beth_{\omega}(\kappa)$ is tight is:

Conclusion 1.2. We have $(A) \Longleftrightarrow(B) \Longleftrightarrow(C)$ where: 
(A) For every $n$ in some forcing extension of $\mathbf{V}$ there are $\kappa, \partial=\beth_{n}(\kappa)$, $\Upsilon>\partial$ and a $\kappa$-family $\mathcal{A} \subseteq[\Upsilon]^{\partial}$ of cardinality $>\Upsilon$.

(B) For every $n$ in some forcing extension of $\mathbf{V}$ there are $\kappa, \partial=\beth_{n}(\kappa)$ and a set $\mathfrak{a}$ of $\partial$ finite sets of regular cardinals $\partial$ such that $J_{<\sup (\mathfrak{a})}[\mathfrak{a}] \subseteq$ $[\mathfrak{a}]^{<\kappa}$, i.e. $\mathfrak{b} \in[\mathfrak{a}]^{\kappa}$ implies that $\max \operatorname{pcf}(\mathfrak{b}) \geq \sup (\mathfrak{a})$.

(C) For every $n$ in some forcing extension of $\mathbf{V}$ there are $\kappa, \partial==\beth_{n}(\kappa)$ and a graph $G$ with list-chromatic number $\kappa$ and coloring number $>\partial$.

Proof of 1.2. $(A) \Longrightarrow(B)$ follows from [11], 6.1 (and $(B) \Longrightarrow(A)$ is obvious by $(*)_{2}$ above).

$(A) \Longrightarrow(C)$ is done in Theorem 2.1 below, where we start letting $\theta=2^{\kappa}$, or, if $\kappa$ is regular, also $\theta=2^{<\kappa}$ suffices to get the assumptions of 2.1. For every $n,(A)_{n} \Longleftrightarrow(B)_{n}$ and $(A)_{2 n+2} \Rightarrow\left(B_{n}\right) \Rightarrow(C)_{n}$.

To prove $(C) \Longrightarrow(B)$ it suffices to note, (use $\left.\theta=\theta^{<\theta}\right)$ that $(a)_{\lambda, \theta, \kappa} \Rightarrow$ $(b)_{\lambda . \theta, \kappa}$ in Claim 2.13. See [8] and use the proof of compactness in singulars [10] and [15], Section 2.

In conclusion, the upper bound $\beth_{\omega}(\kappa)$ cannot be lowered without making substantial progress in pcf theory. If, on the other hand, the negations of $\oplus_{m}^{2}$ are consistent for all $m$, then Kojman's $\beth_{\omega}(\kappa)$ upper bound is optimal.

1.2. Should we expect consistency or better pcf theorems? Let us mention first the known consistency results. Only quite recently Gitik [4] succeeded to prove, from the consistency of large cardinal axioms, the consistency of a countable set of regular cardinals $\mathfrak{a}$ with $\operatorname{pcf}(\mathfrak{a})$ uncountable, but really just $|\operatorname{pcf}(\mathfrak{a})|=\aleph_{1}$. In particular he got $(\mathrm{st})_{\aleph_{0}, \aleph_{1}, \mu}^{2}$. While a great achievement, this is still very distant from what we need.

For $\kappa>\aleph_{0}$ there are no known consistency results. After the RGCH was proved in the early nineties much effort (at least by the present author) was made to lower $\beth_{\omega}$ - so far without any success. However in some other directions there were advances $([16,17,5])$.

So do we expect consistency or ZFC results? Wishful thinking, or, if you prefer, the belief that "set theory behaves in an interesting way" suggests that the answer to "for which $m$ the statement $\oplus_{m}^{1}$ holds in ZFC" should turn out to be somewhere in the middle, e.g. $m=4$ (or $m=957$, for that matter). More seriously, the situation is wide open. Perhaps, as on the one hand the ZFC $\beth_{\omega}(\kappa)$ gap has not changed for a long time now, while on the other hand there has been a recent breakthrough in consistency, there is some sense in viewing consistency as more likely.

\section{PROOFS}

Theorem 2.1. Suppose that $\kappa<\theta=\theta^{<\kappa}, \Upsilon>\partial=\beth_{2 \ell+1}(\theta)$ and there a $\kappa$-family $\mathcal{A} \subseteq[\Upsilon]^{2}$ of size $\Upsilon^{+}$Then in some forcing extension there is a graph $G$ with list-chromatic number $\theta$ and coloring number $>\beth_{\ell}(\theta)$. 
As promised in the introduction, we may prove a weak version of the $\mathrm{RGCH}$ theorem from a bound on list-chromatic number.

Theorem 2.2. Suppose that $\kappa<\theta=\theta^{<\kappa}, \Upsilon>\partial>\beth_{\omega}(\theta)$ and there is a $\kappa$-family $\mathcal{A} \subseteq[\Upsilon]^{\partial}$ of size $\partial^{+}$. Then in some forcing extension there is a graph $G$ with list-chromatic number $\theta$ and coloring number $\geq \partial>\beth_{\omega}(\theta)$.

Convention: For this section we fix $\aleph_{0} \leq \kappa<\theta$.

We shall need the following definition from [14] p. 5. (See also [18] for more on this and other forcing axioms). We recall one implication from 2.4 below: if $\mathcal{A}$ is as in 2.1 then $G_{\mathcal{A}}$, define in 2.4, has coloring number $>\partial$.

Definition 2.3. A forcing notion $\mathbb{P}$ satisfies $*_{\mu}^{\omega}$ where $\aleph_{0}<\mu=\operatorname{cf}(\mu)$ if Player I (the "completeness" player) has a winning strategy in the following game in $\omega$ moves:

At step $k:$ If $k \neq 0$ then Player I chooses $\left\langle p_{1, \alpha}^{k}: \alpha<\mu^{+}\right\rangle$with $p_{1, \alpha}^{k} \in \mathbb{P}$ such that for all $\xi<\zeta$ and for club-many $\alpha<\mu^{+}$in $S_{\mu}^{\mu^{+}}, p_{2, \alpha}^{k-1} \leq p_{1, \alpha}^{k}$, and also chooses a function $f_{k}: \mu^{+} \rightarrow \mu^{+}$which is regressive on a club of $\mu^{+}$. If $k=0$ Player I chooses $p_{1, \alpha i}^{0}=\emptyset_{\mathbb{P}}$ and $f^{0}$ as the identically 0 function on $\mu^{+}$.

Player II chooses $\left\langle p_{2, i}^{k}: i<\mu^{+}\right\rangle$such that for club many $\alpha<\mu^{+}$in $S_{\mu}^{\mu^{+}}$ it holds that $p_{1, \alpha}^{k} \leq p_{2, \alpha}^{k}$.

Player I wins play of if there is a club $E \subseteq \mu^{+}$such that for all $\alpha<\beta$ in $E \cap S_{\mu}^{\mu^{+}}$, if $f_{k}(\alpha)=f_{k}(\beta)$ for all $k<\omega$ then there is an upper bound in $\mathbb{P}$ to the set $\left\{p_{1 . \alpha}^{k}: k<\omega\right\} \cup\left\{p_{2, \beta}^{k}: k<\omega\right\}$.

Definition 2.4. (1) $\mathcal{A}$ is a $\kappa$-family of sets when $|A \cap B|<\kappa$ for all distinct $A, B \in \mathcal{A}$ and is a $(\theta, \kappa)$-family if in addition, $|A| \geq \theta$ for all $A \in \mathcal{A}$ and, for notational transparency, $\mathcal{A} \cap \cup \mathcal{A}=\emptyset$.

(2) Suppose $\mathcal{A}$ is a $\kappa$-family of sets. We define the (bipartite) graph $G_{\mathcal{A}}$. Its set of vertices is $V_{\mathcal{A}}=\mathcal{A} \cup \cup \mathcal{A}$. We denote $\bigcup \mathcal{A}$ by $p t(\mathcal{A})$. The edge set $E_{\mathcal{A}}$ is $\{\{v, A\}: v \in A \in \mathcal{A}\}$. When $\mathcal{A}$ is fixed or clear from context, we refer to $G_{\mathcal{A}}$ as $\langle V, E\rangle$.

Definition 2.5. For $a(\theta, \kappa)$-family $\mathcal{A}$, a set $Y \subseteq G_{\mathcal{A}}$ is closed (pedantically, $\mathcal{A}$-closed, but the identity of $\mathcal{A}$ is clear from the context) if:

(a) $A \neq B \in Y \cap \mathcal{A} \Rightarrow A \cap B \subseteq Y$.

(b) If $A \in \mathcal{A}$ and $|A \cap Y| \geq \kappa$ then $A \in Y$.

A sub-graph $G^{\prime}$ of $G_{\mathcal{A}}$ is closed if its set of vertices is closed.

Claim 2.6. If $\mathcal{A}$ is a $(\theta, \kappa)$-family and $\lambda^{\kappa}=\lambda \geq \theta$ then every sub-graph of $G_{\mathcal{A}}$ of size $\lambda$ is contained in a closed sub-graph of the same size. Moreover, if $Y_{1} \subseteq G$ is closed and $X \subseteq Y_{1}$ is of size $\lambda$, then there is a closed $Y \subseteq Y_{1}$ of cardinality $\lambda$ such that $X \subseteq Y$.

Remark: instead of $\lambda^{\kappa}=\lambda$ it suffices that $\mathcal{D}(\lambda, \kappa)=\lambda$, where $\mathcal{D}(\lambda, \kappa)=$ $\operatorname{cf}\left([\lambda]^{\kappa}, \supseteq\right)$, (see $\left.[7]\right)$. 
Definition 2.7. Suppose $\theta>\kappa$ and $\mu$ are cardinals and $|\alpha|^{\kappa}<\mu$ for all $\alpha<\mu$. We say that $\operatorname{Pr}_{\theta, \kappa}(\mu)$ holds when for every $(\theta, \kappa)$-family $\mathcal{A}$ and every [closed] $Y \subseteq G_{\mathcal{A}}$ of cardinality $|Y|<\mu$ the list chromatic number of $Y$ is at most $\theta$; that is, for every assignments of lists $L(v)$ to vertices in $G_{\mathcal{A}}$ such that $|L(v)| \geq \theta$ there is a valid coloring $c \in \prod_{v} L(v)$.

Claim 2.8. Assume that $\mathcal{A}$ is a $(\theta, \kappa)$-family, $Y \subseteq G_{\mathcal{A}}$ is closed, $\operatorname{cf}(\delta) \neq$ $\operatorname{cf}(\kappa), \delta<\operatorname{cf}(\mu)$ and $Z_{i} \in[Y]^{<\mu}$ is increasing with $i<\delta$. If each $Z_{i}$ is $\mathcal{A}$-closed then $Z:=\bigcup_{i<\delta} Z_{i}$ is $\mathcal{A}$-closed.

Proof. First, if $A \neq B \in Z \cap \mathcal{A}$ then for some $i<\delta$ it holds that $A, B \in Z_{i}$, but $Z_{i}$ is $\mathcal{A}$-closed hence $A \cap B \subseteq Z_{i} \subseteq Z$ as required (in 2.5(a)). Second, if $A \in \mathcal{A}$ satisfies that $|A \cap Z| \geq \kappa$ then (because $\operatorname{cf}(\delta) \neq \operatorname{cf}(\kappa)$ ) for some $i<\delta$ it holds that $\left|A \cap Z_{i}\right| \geq \kappa$ and as $Z_{i}$ is closed, $A \in Z_{i} \subseteq Z$.

Lemma 2.9 (Step-up Lemma). Suppose that $\lambda>\mu=\mu^{<\mu}>\kappa$ and $\theta>\kappa$. Assume that

(a) The internal forcing axiom for posets that satisfy $*_{\mu}^{\omega}$ (see Def 2.3) holds for $<\lambda$ dense sets.

(b) $(\forall \alpha<\mu)\left(|\alpha|^{\kappa}<\mu\right)$.

(c) $(\forall \alpha<\lambda)\left(|\alpha|^{\kappa}<\lambda\right)$

(d) $\operatorname{Pr}_{\theta, \kappa}(\mu)$ holds.

Then $\operatorname{Pr}_{\theta, \kappa}(\lambda)$.

Proof. Suppose

$(*)_{1} \mathcal{A}$ is a $(\theta, \kappa)$-family and $Y_{*} \subseteq G_{\mathcal{A}}$ is closed, $\left|Y_{*}\right|<\lambda$ and $L(v)$ such that $|L(v)|=\theta$ is given for all $v \in Y_{*}$.

We need to prove the existence of a valid coloring $c$ of $G$ such that $c(v) \in$ $L(v)$ for all $v \in Y_{*}$.

Let $\mathbb{P}$ be the following poset: $q \in \mathbb{P}$ iff $q$ is a partial valid coloring for the given lists and $\operatorname{dom}(q) \subseteq Y_{*} \subseteq G_{\mathcal{A}}$ is closed of cardinality $<\mu$. A condition $q$ is stronger than a condition $p, q \geq p$, iff $p \subseteq q$.

$(*)_{2} \mathbb{P}$ is a forcing notion.

$(*)_{3}$ (Density) if $p \in \mathbb{P}$ and $Z \subseteq Y_{*}$ satisfies $|Z|<\mu$ then there is $q \geq p$ such that $Z \subseteq \operatorname{dom}(q)$.

Proof of $(*)_{3}$ : By possibly increasing $Z$, we may assume that $Z$ is closed in $Y_{*}$ and that $\operatorname{dom}(p) \subseteq Z$. As $\operatorname{dom}(p)$ is closed, for all $A \in \mathcal{A} \cap Z \backslash \operatorname{dom}(p)$ it holds that $|A \cap \operatorname{dom}(p)|<\kappa$. For $A \in \mathcal{A} \cap Z \backslash \operatorname{dom}(p)$ let $L^{\prime}(A)=$ $L(A) \backslash\{p(v): v \in A \cap \operatorname{dom}(p)\}$. As $|A \cap \operatorname{dom} p|<\kappa<\theta$ it holds that $\left|L^{\prime}(A)\right|=\theta$.

For $v \in(Z \backslash \operatorname{dom}(p)) \cap p t(\mathcal{A})$ there is at most one $A \in \operatorname{dom}(p)$ such that $v \in A$, because $\operatorname{dom}(p)$ is closed. Let $L^{\prime}(v)$ be gotten from $L(v)$ by subtracting $\{p(A)\}$ from $L(v)$ for that unique $A$, when $A$ exists. For all $x \in \operatorname{dom}(p)$ let $L^{\prime}(x)=L(x)$.

By $\operatorname{Pr}_{\theta, \kappa}(\mu)$ there is condition $p^{\prime \prime}$ with $\operatorname{dom}\left(p^{\prime \prime}\right)=Z$ such that $p^{\prime \prime}(v) \in$ $L^{\prime}(v)$ for every $v \in Z$. Let $p^{\prime}=p^{\prime \prime} \uparrow(Z \backslash \operatorname{dom}(p))$ and let $q=p \cup p^{\prime}$. Now 
we claim that $q \in \mathbb{P}$. As $x \in Z \Rightarrow q(x) \in L^{\prime}(x) \subseteq L(x)$ for all $x \in \operatorname{dom}(q)$, all that needs to be checked is the validity of the coloring $q$. Suppose that $v \in A$ and $v, A \in \operatorname{dom}(q)$. First assume that $v \in \operatorname{dom}(p)$ and $A \in \operatorname{dom}\left(p^{\prime}\right)$. In this case $p(v) \neq p^{\prime}(A)$ because $p(v) \notin L^{\prime}(A)$ by the definition of $L^{\prime}(A)$. Another case to check is $v \in \operatorname{dom}\left(p^{\prime}\right)$ and $A \in \operatorname{dom}(p)$, which followed from the choice of $L^{\prime}(v)$. The two remaining cases are clear, holds as $p, p \in \mathbb{P}$.

$(*)_{4}$ If $\left\langle p_{i}: i<\delta\right\rangle$ is an increasing sequence of conditions in $\mathbb{P}$ and $\delta<\mu$ and $\operatorname{cf}(\delta) \neq \operatorname{cf}(\kappa)$ then the union is a condition, and is an upper bound of the sequence.

Proof of $(*)_{4}$ : Let $\left.Y_{\delta}=\bigcup\left\{\operatorname{dom} p_{i}\right): i<\delta\right\}$. Now $\left|Y_{\delta}\right|<\mu$ as $\delta<\mu$ by the assumptions, and $i<\delta \Rightarrow \operatorname{dom}\left(p_{i}\right) \in\left[Y_{*}\right]^{<\mu} \subseteq\left[G_{\mathcal{A}}\right]^{<\mu}$ by the definition of $\mathbb{P}$, recalling that $\mu$ is regular (by $\mu=\mu^{<\mu}$ from of the claim's assumptions). Since $\operatorname{cf}(\delta) \neq \kappa$, by 2.8 it holds that $p=\bigcup_{i} p_{i}$ is a condition.

$(*)_{5}$ If $\delta<\mu, \bar{p}=\left\langle p_{i}: i<\delta\right\rangle$ is increasing in $\mathbb{P}$ and $\operatorname{cf}(\delta)=\operatorname{cf}(\kappa)$ then $\bar{p}$ has an upper bound in $\mathbb{P}$.

Proof of $(*)_{5}$ : Let $Z \subseteq Y_{*} \subseteq G_{\mathcal{A}}$ be closed such that $|Z|<\mu$ and $Y=$ $\bigcup_{i<\delta} \operatorname{dom}\left(p_{i}\right) \subseteq Z$. By restricting to a subsequence we may assume that $\delta=\operatorname{cf}(\kappa)$ and so $A \in \mathcal{A} \backslash Y \Rightarrow \bigwedge_{i<\kappa}\left(\left|A \cap \operatorname{dom}\left(p_{i}\right)\right|<\kappa \Rightarrow|A \cap Y| \leq \kappa\right.$. Now repeat the proof of $(*)_{3}$ with $p=\bigcup_{i} p_{i}$ with the following changes:

(a) if $A \in Z \backslash Y, A \in \mathcal{A}$, then $|A \cap Y| \leq \kappa$ hence $L^{\prime}(A)=L(A) \backslash\{p(v)$ : $v \in A \cap Y\}$ has cardinality $\theta$ as $L(A)$ has cardinality $\theta>\kappa \geq$ $|A \cap Y| \geq|\{p(v): v \in A \cap Y\}|$.

(b) if $v \in Z \backslash Y, v \in p t\left(G_{\mathcal{A}}\right)$, then

$$
\left.i<\kappa \Rightarrow \mid\left\{A \in \operatorname{dom}\left(p_{i}\right) \cap\right] \mathcal{A}: v \in A\right\} \mid \leq 1,
$$

hence

$$
|\{A \in \mathcal{A} \cap Y: v \in A\}| \leq 1
$$

and $L^{\prime}(v)=L(v) \backslash\left\{p(A): A \in Y_{\delta} \wedge v \in A\right\}$ has cardinality $\theta$.

Now we can finish as in $(*)_{3}$.

$(*)_{6}\left\{p_{\zeta}^{\ell}: \ell=1,2\right.$ and $\left.\zeta<\delta\right\}$ has a common upper bound when:

(a) $\delta<\kappa^{+} \leq \mu$ (we will use $\delta=\omega<\kappa^{+}$when simpler as this us the one we shall use). 2016-02-09 simpler).

(b) $p_{\zeta}^{\ell} \in \mathbb{P}$

(c) $\zeta<\xi<\delta \Rightarrow p_{\zeta}^{\ell} \leq_{\mathbb{P}} p_{\xi}^{\ell}$.

(d) $p_{\zeta}^{1}, p_{\zeta}^{2}$ are compatible functions for $\zeta<\delta$.

Proof of $(*)_{6}$ : Let $p=\bigcup_{\ell, \zeta} p_{\zeta}^{\ell}$, so $p$ is a function, but not necessarily a condition in $\mathbb{P}$. Let $Y=\operatorname{dom}(p)$ and $Z \supseteq Y$ be closed and of cardinality $<\mu$ such that $Z \subseteq Y_{*} \subseteq G_{\mathcal{A}}$.

(A) If $A \in Z \backslash Y, \bar{A} \in \mathcal{A}$, and $\ell \in\{1,2\}$ then $\ell \in\{1,2\} \wedge \zeta<\delta \Rightarrow$ $\left|A \cap \operatorname{dom}\left(p_{\zeta}^{\ell}\right)\right|<\kappa$ so $\left\langle\left|A \cap \operatorname{dom}\left(p_{\zeta}^{\ell}\right)\right|: \zeta<\delta\right\rangle$ is a non-decreasing sequence of sets each of cardinality $<\kappa$ hence $\leq \kappa$. So $\left|A \cup \bigcup_{\zeta} \operatorname{dom}\left(p_{\zeta}^{\ell}\right)\right| \leq \kappa$, hence $|A \cap Y| \leq \kappa$. 
(B) If $v \in Z \backslash Y, v \in p t\left(G_{\mathcal{A}}\right)$, and $\ell \in\{1,2\}$ then $\mid\left\{A \in \bigcup_{\zeta} \operatorname{dom}\left(p_{\zeta}^{\ell}\right), v \in\right.$ $A\} \mid \leq 1$ hence $|\{A \in Z \backslash A: A \in \mathcal{A}, v \in A\}| \leq 2$. So all is fine.

We continue as in the proof of $(*)_{5}$.

$(*)_{7} \mathbb{P}$ is $\mu$-complete (by $\left.(*)_{4}+(*)_{5}\right)$.

$(*)_{8}$ The property $*_{\mu}^{\omega}$ holds for $\mathbb{P}$.

The game which defines $*_{\mu}^{\omega}$ lasts $\omega$ steps and at each step $k<\omega$ for $\ell=1,2$ a sequence of conditions $\left\langle p_{1, \alpha}^{k}: \alpha<\mu^{+}\right\rangle$, a club $E_{k} \subseteq \mu^{+}$and a regressive function $f_{k}$ with domain $\mu^{+}$played by the completeness player I (see Def 2.3 or [14] p. 5. See also [18] for more on this and related forcing axioms).

This is how player I chooses $E_{k}$ and $f_{k}: E_{k}$ is sufficiently closed; $f_{k}$ has domain $\mu^{+}$and is regressive such that:

$\oplus$ If $\alpha_{1}, \alpha_{2} \in \operatorname{dom}\left(f_{k}\right) \cap S_{\mu}^{\mu^{+}} \cap E_{k}, f_{k}\left(\alpha_{1}\right)=f_{\zeta}\left(\alpha_{2}\right)$ then $p_{1, \alpha_{1}}^{k}, p_{1, a_{2}}^{k}$ are compatible functions.

This clearly suffices (as the $\left\langle\left(p_{1, \alpha_{1}}^{k}, p_{1, \alpha_{2}}^{k}\right): k<\delta\right\rangle$ are like $\left\langle\left(p_{\zeta}^{1}, p_{\zeta}^{2}\right)\right.$ in $\left.(*)_{6}\right)$.

Clearly such a function $f_{k}$ exists but we elaborate.

$(*)_{8.1} f_{\zeta}(\delta)$ codes:

(a) $a_{\delta}^{k}=\operatorname{dom}\left(p_{1, \delta}^{k}\right) \cap\left(\bigcup_{\alpha<\delta} \operatorname{dom} p_{1, \alpha}^{k}\right)$.

(b) $p_{1, \delta}^{k}\left\lceil a_{\delta}^{k}\right.$.

(c) $\left\{p_{1, \delta}^{k}(v): v \in \operatorname{dom}\left(p_{1, \delta}^{k}\right) \cap \cap(\mathcal{A})\right.$ and $\left.p_{1, \delta}^{k}(v) \in \bigcup_{\alpha<\delta} \operatorname{Rang}\left(p_{1, \alpha}^{k}\right)\right\}$

(d) $\left\{p_{1, \delta}^{k}(A): A \in \operatorname{dom}\left(p_{1, \delta}^{k}\right) \cap \mathcal{A}\right.$ and $\left.p_{1, \delta}^{k}(A) \in \bigcup_{\alpha<\delta} \operatorname{Rang}\left(p_{1, \alpha}^{k}\right)\right\}$

[ What is the point of clauses (c), (d)? Consider $p_{1, \alpha_{1}}^{k}, p_{1, \alpha_{2}}^{k}$ with $\alpha_{1}<\alpha_{2}$; maybe there are $\ell \in\{1,2\}, v \in \operatorname{dom}\left(p_{1, \alpha_{\ell}}^{k}\right) \cap p t\left(G_{\mathcal{A}}\right), A \in \operatorname{dom}\left(p_{1, \alpha_{3-\ell}}^{k}\right) \cap \mathcal{A}$ such that $v \in A$ and $p_{1, \alpha_{\ell}}^{k}(v)=p_{1, \alpha_{3-\ell}}^{k}(A)$. Those are avoided by those clauses. 2016-02-10 s

So now player I wins as whenever $\alpha<\beta$ belong to $S_{\mu}^{\mu^{+}} \cap \bigcap_{k} E_{k}$ and $\bigwedge_{k} f_{k}(\alpha)=f_{k}(\beta)$, the set of conditions $\left\{p_{1, \alpha}^{k}: k<\omega\right\} \cup\left\{p_{1, \beta}^{k}: k<\omega\right\}$ has an upper bound in $\mathbb{P}$ by $(*)_{6}$.

This proves $(*)_{8}$.

$(*)_{9}$ If $x \in Y_{*}$ then $D_{x}$ is an open dense subset of $\mathbb{P}$ where

$D_{x}=\{p \in \mathbb{P}: x \in \operatorname{dom}(p)\}$

[ Why $(*)_{9}$ holds? by $(*)_{3}$.]

By the axiom for posets with $*_{\mu}^{\omega}$, there is a generic filter for $\mathbb{P}$ which meets all dense sets $D_{x}$ for $x \in Y$, where $p \in D_{x}$ if $x \in \operatorname{dom}(p)$. The union of the generic is a valid coloring from the lists on $Y_{*}$.

Corollary 2.10. Suppose $n \geq 1$ and

(a) $\mu_{0}<\mu_{1} \cdots<\mu_{n}$.

(b) For all $l \leq n-1$ it holds that $\left(\forall \alpha<\mu_{\ell}\right)\left(|\alpha|^{\kappa}<\mu_{\ell}\right)$.

(c) $\mu_{\ell}^{<\mu_{\ell}}=\mu_{\ell}$ and $2^{\mu_{i}}=\mu_{i+1}$ for $i<n$. 
(d) For every $\ell<n$, the forcing axiom for posets with $*_{\mu_{\ell}}^{\omega}$ and $<\mu_{\ell+1}$ dense sets holds.

(e) $\mu_{0} \leq \theta^{+}$and $\kappa<\theta$.

Then $\operatorname{Pr}_{\theta, \kappa}\left(\mu_{n}\right)$.

Proof. By induction on $n$. Since the list-chromatic number of any graph $G$ of cardinality $<\mu_{0}$ is $\leq|G| \leq \theta$, the condition $\operatorname{Pr}_{\theta, \kappa}\left(\mu_{0}\right)$ holds trivially. The induction step follows from the main lemma 2.9.

Next we show how to force the conditions of the previous lemma.

Claim 2.11. Assume that:

(A) $\theta=\theta^{<\kappa}>\kappa$ and $\beth_{2 n+1}(\theta)<\mu \leq \chi<\lambda$.

(B) $(s t)_{\kappa, \mu, \chi, \lambda}^{1}$.

Then: For some $\mathbb{P}$ and $\bar{\mu}$

(a) $\mathbb{P}$ is a $\theta^{+}$-complete forcing notion that satisfies $\left(\beth_{2 n+1}(\theta)\right)^{+}$-c.c.

(b) $\bar{\mu}=\left\langle\mu_{\ell}: \ell \leq n\right\rangle$ in $\mathbf{V}^{\mathbb{P}}$ such that $\mu_{\ell}=\mu^{\mu_{\ell}}=\left(\beth_{\ell}(\theta)\right)^{+}<\beth_{\ell+1}(\theta)$ for all $\ell \leq n,|\alpha|^{\kappa}<\mu_{\ell}$ for all $\alpha<\mu_{\ell}$ and $\beth_{n}(\theta)<\mu \leq \chi<\beth_{n+1}(\theta)$.

(c) $(\mathrm{st})_{\kappa, \mu, \chi, \lambda}^{1}$.

(d) The forcing axiom $*_{\mu_{\ell}}^{\omega}$ with $<\mu_{\ell+1}$ holds for all $\ell \leq n$.

Proof. Now,

$(*)_{1}\left(\beth_{2 n+1}(\theta)\right)^{+} \leq \partial$ and $\left(\beth_{2 n+1}(\theta)\right)^{+}<\Upsilon$

Why $(*)_{1}$ ? The first inequality hold by $(\mathrm{A})$. For the second, letting $\chi_{1}=$ $\beth_{2 n+1}(\theta)^{+}$we have $\chi_{1}^{\beth_{2 n}(\theta)}=\chi_{1}$ hence $\chi_{1}^{\kappa}=\chi_{1}$ whereas $\Upsilon^{\kappa} \geq \lambda>\mu$ because we are assuming $(\mathrm{st})_{\kappa, \mu, \chi, \lambda}^{1}$

Now let

$(*)_{2} \quad$ (a) $\mu_{\ell}=\left(\beth_{2 \ell}(\theta)\right)^{+}$for $\dagger \ell \leq n$ so $2^{<\mu_{\ell}} \leq \beth_{2 \ell+1}(\theta)$.

(b) Choose $\mu_{n+1}$ such that $\mu_{n+1}=\operatorname{cf}\left(\mu_{n+1}\right)=\left(\mu_{n+1}\right)^{\left(\mu_{n}^{\kappa}\right)}>\lambda \mathrm{s}$ and $\alpha<\mu_{n+1} \Rightarrow|\alpha|^{\kappa}<\mu_{n+1}$

Remark: less suffices; $\mu_{n+1}=\left(\lambda^{\kappa}\right)^{+}$or just $\mu_{n+1}=\operatorname{cf}\left(\mu_{n+1}\right)>\lambda$ satisfies $\left(\forall \alpha<\mu_{n+1}\right)\left(|\alpha|^{\kappa}<\mu_{n+1}\right)$, but will complicate the notation below, e.g. $(*)_{4}(b)$ for $\ell=n$ is different.

Now

$(*)_{3} \quad$ (a) $\mu_{0}=\theta^{+}$hence $\mu_{0}=\operatorname{cf}\left(\mu_{0}\right)$ and $(\forall \alpha)\left(\alpha<\mu_{0} \rightarrow|\alpha|^{\kappa} \leq \theta^{\kappa}=\right.$ $\left.\theta<\mu_{0}\right)$.

(b) $\mu_{0}<\mu_{1}<\cdots<\mu_{n}<\mu_{n+1}$ are regular.

(c) $\left(\forall \alpha<\mu_{\ell}\right)\left(|\alpha|^{\kappa}<\mu_{\ell}\right)$ for all $\ell \leq n+1$.

(d) $\left(\mu_{\ell+1}\right)^{2^{<\mu_{\ell}}}=\mu_{\ell+1}$.

(e) $\mu_{n}<\mu \leq \chi<\lambda<\mu_{n+1}$.

Let

$(*)_{4}$ Let

(a) $\mathbb{Q}_{\ell}^{*}=\operatorname{Levy}\left(\mu_{\ell}, 2^{<\mu_{\ell}}\right)$ for $\ell \leq n$.

(b) $\mathbb{Q}_{*}=\prod_{\ell \leq n} \mathbb{Q}_{\ell}^{*}$. 
(c) $Q_{\leq k}^{*}=\prod_{\ell \leq k} \mathbb{Q}_{\ell}^{*}$

Easily,

$(*)_{5} \quad$ (a) $\mathbb{Q}_{\ell}^{*}$ is $\mu_{\ell^{-}}$-complete and of cardinality $2^{<\mu_{\ell}}$. so satisfies the $2^{<\mu_{\ell}}$ cc.

(b) Let $\mathbf{V}_{\ell}:=\mathbf{V}^{\prod_{k<\ell} \mathbb{Q}_{\kappa}^{*}}$

$(*)_{6}$ in $\mathbf{V}_{n+1}:=\mathbf{V}^{\prod_{\ell \leq n} \mathbb{Q}_{\ell}}$, we define $\left\langle\left(\mathbb{P}_{k}, \underset{\sim}{\mathbb{Q}_{\ell}^{2}}\right): k \leq n+1, \ell \leq n\right\rangle$ such that :

(a) $\mathbb{P}_{0}$ is the trivial forcing.

(b) $\mathbb{P}_{\ell+1}$ is a forcing notion of cardinality $\mu_{\ell+1}$.

(c) $\mathbb{P}_{\ell+1}$ satisfies the $\mu_{\ell}^{+}$-c.c.

(d) $\mathbb{P}_{\ell+1}=\mathbb{P}_{\ell} * \underset{\sim}{\mathbb{Q}_{\ell}^{2}}$.

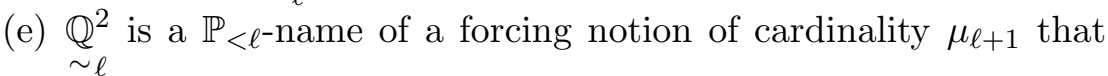
satisfies $\mu_{\ell}^{+}$-c.c. that forces $2^{\mu_{\ell}}=\mu_{\ell+1}$ and the axiom for forcing notions that satisfy $*_{\mu_{\ell}}^{\omega}$ for $<\min \left\{\mu_{\ell+1},\left(\partial^{\kappa}\right)^{+}\right\}$dense sets.

(f) $\mathbb{P}_{k+1}$ is a $\mathbb{Q}_{*}$-name and actually a $\mathbb{Q}_{\leq k}^{*}$-name

There is no problem to carry the induction (note that $\left(\mu_{\ell+1}\right)^{<\mu}=\mu_{\ell+1}$ in $\mathbf{V}_{n+1}^{\mathbb{P}_{n}+1}$.) We return to $\mathbf{V}$, so we have a $\underset{\mathbb{Q}}{\mathbb{Q}}$-names so $\underset{\sim}{\mathbb{P}}$ for $\ell=0, \ldots, k+1$ for the forcing notion above. Let, in $\mathbf{V}, \mathbb{P}^{k+1}=\mathbb{Q}_{\leq k}^{*} * \underset{\sim}{\mathbb{P}}$. Why $\mathbb{P}=\mathbb{P}^{n}$ is as required?

Clearly, all forcing notions $\mathbb{Q}_{\ell}^{*}, \mathbb{Q}_{*}, \mathbb{Q}_{\leq k}^{*}, \mathbb{P}^{k}$, are $\theta^{+}$-complete, hence in particular so is $\mathbb{P}$. Therefore, in $\mathbf{V}^{\mathbb{P}}$ still $\left(\forall \alpha<\mu_{\ell}\right)\left(|\alpha|^{\kappa}<\mu_{\ell+1}\right)$ for all $\ell<n+1$ because we prove below that $\mu_{\ell}$ does not collapse.

Clearly, $\mathbb{P}^{k+1}$ has cardinality $\mu_{k+1}$ and satisfies the $\left(2^{<\mu_{k}}\right)^{+}$-cc and in $\mathbf{V}$ the forcing notion $\mathbb{Q}_{\ell}$ are $\mu_{\ell}$-complete and in $\mathbf{V}^{\mathbb{Q}_{\leq k}^{*}}$ the forcing notion $\mathbb{Q}_{\ell}^{2}$ is forced to be $\mu_{\ell}$-complete. Hence in $\mathbf{V}$ for $\ell \leq k$ we have $\vdash_{\mathbb{P}^{k}} " \mu_{\ell}=\mu_{\ell}^{<\mu_{\ell}}$ is not collapsed", and $\mathbb{P}^{k}$ satisfies the $\left(\left(2^{<\mu_{k}}\right)^{+}\right)$-c.c. as $\mathbb{Q}_{\leq k}^{*}$ does, and $\mathbb{P}_{k+1}$ satisfies $\left(\mu^{\kappa}\right)+$-c.c..

Lastly, the relevant forcing axiom holds: if $\ell<n$, the one for $(*)_{\mu_{\ell}}^{\varepsilon}$ and $<\mu_{\ell+1}$-dense sets. So replacing $\mu_{n+1}$ by $\left(\mu^{\kappa}\right)^{+}$and applying 2.6 we are done.

A similar argument works to replace $n$ with $\omega$ :

Theorem 2.12. The condition $(A)_{\ell(*)}$ implies the condition $(B)_{\ell(*)}$ for $\ell(*) \in\{1,2\}$, where:

$(A)_{1} \aleph_{0}<\operatorname{cf}(\kappa) \leq \kappa<\theta=\theta^{<\kappa}, \chi \geq \lambda \geq \beth_{\omega}(\kappa)$ and there exists $a$ $\kappa$-family $\mathcal{A} \subseteq[\chi]^{\lambda}$ of cardinality $|\mathcal{A}| \geq \chi^{+}$.

$(A)_{2} \aleph_{0}<\operatorname{cf}(\kappa) \leq \kappa<\theta=\theta^{<\kappa}$ and for every $n<\omega$ there are $\chi_{n}>$ $\lambda_{n} \geq \beth_{n}(\theta)$ a $\kappa$-family $\mathcal{A}_{n} \subseteq\left[\chi_{n}\right]^{\lambda_{n}}$ of cardinality $\left|\mathcal{A}_{n}\right| \geq \chi_{n}^{+}$and $\lambda_{n} \notin\left[\beth_{\omega}(\theta), \beth_{\theta+1}(\theta)\right]$ 
$(B)_{1}$ For some forcing notion $\mathbb{P}$ not adding new sequences of ordinals of length $<\theta$, it holds that:

- $\left(\beth_{\omega}(\theta)\right)^{\mathbf{V}^{\mathbb{P}}}=\left(\beth_{\omega}(\theta)\right)^{\mathbf{V}}$.

- There exists a graph $G$ with list-chromatic number $\theta$ and coloring number $>\left(\beth_{\omega}(\theta)\right)^{+}$.

$(B)_{2}$ Like $(B)_{1}$ with the coloring number $\geq\left(\beth_{\omega}(\theta)\right)^{+}$.

Proof. Stage A. For $(A)_{1} \Rightarrow(B)_{1}$ assume $(A)_{1}$ and let

$(*)_{1}\left(\chi_{n}, \lambda_{n}\right)=(\chi, \lambda), \mathcal{A}_{n}=\mathcal{A}$, so we can assume $(A)_{2}$.

$(*)_{2}$ Let $u_{1}=\left\{n: \lambda_{n}<\beth_{\omega}(\theta)\right\}$ hence $\left.n \in u_{1} \Rightarrow \lambda_{n}<\beth_{\omega}\right) \theta$ ) and let $u_{3}=\left\{n: \lambda_{n}>\beth_{\omega}(\theta)\right\}$.

Recalling clause $(A)_{2}$ note that $u_{1}, u_{3}$ is a partition of $\omega$.

$(*)_{3}$ Without loss of generality, for some $\mathbf{i} \in\{1,2,3\}$ we have:

(a) $u_{\mathbf{i}}=\omega$.

(b) If $\mathbf{i}=3$ without loss of generality there is some $\lambda_{*}>\beth_{\omega}(\theta)$ such that $\bigwedge_{n} \lambda_{n}=\lambda_{*}$.

(c) If $\mathbf{i}=2$ let $\mu_{*}=\beth_{\omega}(\theta)$.

Stage B. Now

$(*)_{4}$ Without loss of generality there is a sequence $\left\langle\mu_{n}: n<\omega\right\rangle$ such that

(a) $\mu_{0}=\theta^{+}$.

(b) $\mu_{n}=\operatorname{cf}\left(\mu_{n}\right)$.

(c) $2^{\mu_{n}}=\mu_{n+1}$.

(d) Hence $\sum_{n} \mu_{n}=\beth_{\omega}(\theta)$.

(e) The forcing axiom $*_{\mu_{n}}^{\omega}$ and $<\mu_{n+1}$ dense sets holds.

Why? As in the proof of 2.11, but note that the forcing may (in fact do) collapse $\beth_{\omega+1}$ to $\beth_{\omega}(\omega)^{+}$. Also in the case $\mathbf{i}=1$ letting wlog $\lambda_{n} \in$ $\left[\beth_{k(n)}, \beth_{k(n)+1}(\omega)\right)$ wlog $k(n)$ is increasing and $k(n+1)>k(n)+n$.

$(*)_{5}$ Without loss of generality, in addition, letting $\theta_{\omega}=\left(\beth_{\omega}(\theta)\right)$, we have $2^{\theta_{\omega}}=\theta_{\omega}^{+}$and $\mu_{\omega+1}=2^{\mu_{\omega}}$ is $>\sum_{n} \chi_{n}$ and as in $(*)_{4}(e)$ the forcing axiom $*_{\theta_{\omega}^{+}}^{\omega}$ and $<\mu_{\omega+1}$ dense sets holds.

Stage C. We deal with the case $\mathbf{i}=1$.

By 2.10, for every $n, \operatorname{Pr}_{\theta, \kappa}\left(\mu_{n}\right)$ holds. By easy compactness for singulars argument we have, as $\aleph_{0}<\operatorname{cf}\left(\theta_{*}\right)$, also $\operatorname{Pr}_{\theta, \kappa}\left(\mu_{\omega}\right)$.

Now clearly for each $n, \partial_{n}<\Upsilon_{n}$ as in the proof of Theorem 1 , there is a graph $G_{n}$ with $\left|\mathcal{A}_{n}\right|$ vertices, coloring number $\geq \lambda_{n}$ and list-chromatic number $\theta$.

Taking then the disjoint sum of all $G_{n}$ we have established $(A)_{2} \Rightarrow(B)_{2}$.

Stage D. $\mathbf{i} \in\{3\}$. Similarly, but we use $(*)_{5}$.

Remark: We can replace $\beth_{\omega}(\theta)$ with $\beth_{\delta(*)}(\theta)$ when $\delta(*)<\operatorname{cf}(\kappa)$.

Proof of Theorems 1 and 2. The proofs consists of combining the lemmas above. 
We conclude with a few simple implications that are needed above.

Claim 2.13. Assume that $\theta$ is a regular cardinal and $2^{\kappa} \leq \theta \leq \lambda$. We have $(a)_{\lambda, \theta, \kappa} \Rightarrow(b)_{\lambda, \theta, \kappa} \Rightarrow(c)_{\lambda, \theta, \kappa} \Rightarrow(d)_{\lambda, \theta, \kappa}$. If, in addition, $\theta=\theta^{\kappa}$ (or just $\partial<\theta \Rightarrow \partial^{\kappa}<\lambda$ then $(d)_{\lambda, \theta, \kappa} \Rightarrow(e)_{\lambda, \theta, \kappa} \Rightarrow(f)_{\lambda, \theta, \kappa}$,

Where

$(a)_{\lambda, \theta, \kappa} \lambda$ is minimal such that there is a graph $G$ with $\lambda$ vertices, coloring number $\geq \theta$ and list-chromatic number $\leq \kappa$.

$(b)_{\lambda, \theta, \kappa} \lambda$ is regular and there is a graph $G$ with $\lambda$ vertices, coloring number $\geq \theta$, every sub-graph of $G$ with $<\lambda$ vertices has coloring number $\leq \theta$ and the complete bipartite graph $K\left(\kappa, 2^{\kappa}\right)$ is not weakly embeddable into $G$.

$(c)_{\lambda, \theta, \kappa} \lambda>\theta$ is regular and there is $\bar{C}$ such that:

$(\alpha) \bar{C}=\left\langle C_{\delta}: \delta \in S\right\rangle$

( $\beta) S \subseteq\{\delta: \delta<\lambda \wedge \mathrm{cf}(\delta)=\theta\}$ is stationary.

$(\gamma) C_{\delta} \subseteq \delta$ and otp $\left(C_{\delta}\right)=\theta$

( $\delta)$ If $u \in[\lambda]^{\kappa}$ then $\left\{\delta \in S: u \subseteq C_{\delta}\right\}$ is bounded in $\lambda$.

$(d)_{\lambda, \theta, \kappa} \lambda>\theta$ is regular and for some $\mu<\lambda$ for every $\partial \in[\kappa, \theta)$ there is $\mathcal{A} \subseteq[\mu]^{\partial}$ of cardinality $\lambda$ such that $u \in[\mu]^{\kappa} \Rightarrow\left(\exists^{<\lambda} v \in \mathcal{A}\right)(u \subseteq v)$

$(e)_{\lambda, \theta, \kappa} \lambda>\theta$ is regular and there are $\mu<\lambda$ and $\left\{A_{\partial}: \partial \in[\kappa, \theta)\right\}$ such that $A_{\partial} \subseteq[\mu]^{\partial}$ is a $\kappa$-family of cardinality $\lambda$.

$(f)_{\lambda, \theta, \kappa} \lambda>\theta$ is regular and there are $\mu<\lambda$ and $\left\{\mathfrak{a}_{\partial}: \partial \in[\kappa, \theta)\right\}$ such that $\mathfrak{a} \subseteq \operatorname{Reg} \cap(\mu \backslash \theta),\left|\mathfrak{a}_{\partial}\right|=\partial$ and $\left(\prod \mathfrak{a}_{\partial},<_{\left[\mathfrak{a}_{\partial}\right]<\kappa}\right)$ is $\lambda$-directed.

Proof. $(a)_{\lambda, \theta, \kappa} \Rightarrow(b)_{\lambda, \theta, \kappa}$. Choose $G$ witnessing $(a)_{\lambda, \theta, \kappa}$. We know that $\lambda$ is regular, and without loss of generality the vertex set of the graph is $\lambda$. The coloring number is $\geq \theta$ by the choice of $G$. If $H \subseteq G$ has fewer than $\lambda$ vertices then it has coloring number $<\theta$ by the minimality of $\lambda$. Also the complete bipartite graph $K\left(\kappa, 2^{\kappa}\right)$ and even $K\left(\kappa, \kappa^{+}\right)$is not weakly embedded in $G$ because its list-chromatic number is $\kappa^{+}$and $\lambda>2^{\kappa}$ and even just $\lambda>\kappa^{+}$. Minimality of $\lambda$ gives more. So $(b)_{\lambda, \theta, \kappa}$ holds.

$(b)_{\lambda, \theta, \kappa} \Rightarrow(c)_{\lambda, \theta, \kappa}$. See [9] or [10]. Assume that the vertex set is $\lambda$ and let $S=\{\delta:(\exists \alpha \geq \delta)(|G[\alpha] \cap \delta| \geq \theta\}$ where $G[\alpha]=\{\beta:(\alpha, \beta)$ is an edge of $G\}$. If $S$ is not stationary then using "every subgraph with $<\lambda$ vertices has coloring number $\leq \theta$ " we conclude that $G$ has coloring number $\leq \theta$. By renaming we get $(c)_{\lambda, \theta, \kappa}$.

$(c)_{\lambda, \theta, \kappa} \Rightarrow(d)_{\lambda, \theta, \kappa}$. For each $\partial \in[\kappa, \theta)$ we find, by Fodor's lemma, $\alpha_{\partial}<\lambda$ such that $\mathcal{A}_{\partial}=\left\{\delta \in S:\left|C_{\partial} \cap \alpha_{\partial}\right| \geq \partial\right\}$ has cardinality $\lambda$. So $\alpha_{*}=\bigcup_{\partial} \alpha_{\partial}<\lambda$ satisfies the desired conclusion for the $\mu$ that is defined as $\mu=\left|\alpha_{*}\right|$ so by renaming we are done.

$(d)_{\lambda, \theta, \kappa} \Rightarrow(e)_{\lambda, \theta, \kappa}$. When, e.g., $\partial<\theta \Rightarrow \partial^{\kappa}<\lambda$ for each $\partial \in[\kappa, \theta)$ let $\left\langle u_{\partial, \alpha}: \alpha<\lambda\right\}$ list $\mathcal{A}_{\partial}$, and for $\alpha<\lambda$ let $W_{\alpha}=\left\{\beta<\lambda:\left|u_{\gamma, \beta} \cap u_{\partial, \alpha}\right| \geq \kappa\right\}$. As $\left|\left[u_{\partial, \alpha}\right]^{\kappa}\right| \leq \partial<\lambda=\operatorname{cf}(\lambda)$, the set $W_{\partial}$ is bounded in $\lambda$, hence for some club $E_{\partial} \subseteq \lambda$ it holds that $\alpha<\beta \in E_{\partial} \Rightarrow\left|u_{\gamma, \alpha} \cap u_{\partial, \beta}\right|<\kappa$, so $\left\{u_{\partial, \alpha}: \alpha \in E_{\partial}\right\}$ is as required.

$$
(e)_{\lambda, \theta, \kappa} \Rightarrow(f)_{\lambda, \theta, \kappa} \text { if } \partial<\theta \Rightarrow \partial^{\kappa}<\lambda \text {. By [11] 6.1. }
$$


SAHARON SHELAH

\section{REFERENCES}

[1] N. Alon. Degrees and choice numbers. Random Structures Algorithms 16 (2000), no. 4, 364-368.

[2] P. Erdős and A. Hajnal. On chromatic number of graphs and set systems. Acta Math. Acad. Hung. 17 (1966) 61-99.

[3] P. Erdős, A. L. Rubin and H. Taylor. Choosability in graphs. Proc. West Coast Conf. on Combi- natorics, Graph Theory and Computing, Congressus Numerantium XXVI, 1979, 125-157.

[4] M. Gitik. Short Extenders Forcing II., preprint. http://www.math.tau.ac.il/ gitik/somepapers.html

[5] M. Gitik and S. Shelah. Applications of pcf for mild large cardinals to elementary embeddings . Annals Pure and Applied Logic 164 (2013) 855-865.

[6] P. Komjath. The list chromatic number of infinite graphs. Israel J. Math. (2014) 67-94.

[7] M. Kojman. Shelah's Revised GCH theorem and a question of Alon on Infinite Graphs Colorings. Israel. J. Math. (2014)

[8] S. Lifsches and S. Shelah. Random Graphs in the monadic theory of order. Archive for Math Logic 38 (1999) 273-312.

[9] S. Shelah. A compactness theorem for singular cardinals, free algebras, Whitehead problem and transversals. Israel J Math 21 (1975) 319-349

[10] S. Shelah. Compactness in singular cardinals revisited, 266 in Shelah-s list, preprint. arxiv:math.LO/1401.3175

[11] S. Shelah. More on Cardinal Arithmetic . Archive for Math Logic 32 (1993) 399-428.

[12] S. Shelah. Advances in Cardinal Arithmetic., Finite and Infinite Combinatorics in Sets and Logic NATO Adv. Sci. Inst. Ser. C Math. Phys. Sci., 411, Kluwer Acad. Publ., Dordrecht, 1993. (1993) 355-383

[13] S. Shelah. The Generalized Continuum Hypothesis revisited. Israel J. Math 116 (2000) 285-321.

[14] S. Shelah. Was Sierpiński right? IV. J. Symbolic Logic 65 (2000) 1031-1054.

[15] S. Shelah. Anti-homogeneous Partitions of a Topological Space. Scientiae Math. Japonicae 59, No. 2; (special issue:e9, 449-501) (2004) 203-255.

[16] S. Shelah. Two cardinals models with gap one revisited . Math Logic Quarterly 51 (2005) 437-447.

[17] S. Shelah. pcf and abelian groups. Forum Math. 25(2013)967-1048

[18] S. Shelah. Forcing axioms for $\lambda$-complete $\mu^{+}$-c.c.; 1036 in Shelah-s list; preprint.

Department of Mathematics, Hebrew University of Jerusalem, Jerusalem, 9190401 ISRAEL 